Pacific Journal of Mathematics

REGULARITY OF CAPILLARY SURFACES OVER DOMAINS

LEN 


\title{
REGULARITY OF CAPILLARY SURFACES OVER DOMAINS WITH CORNERS
}

\section{LEON SIMON}

\begin{abstract}
Using the usual mathematical model (capillary surface equation with contact angle boundary condition) we discuss regularity of the equilibrium free surface of a fluid in a cylindrical container in case the container cross-section has corners.

It is shown that good regularity holds at a corner if the "corner angle" $\theta$ satisfies $0<\theta<\pi$ and $\theta+2 \beta>\pi$, where $0<\beta \leq$ $\pi / 2$ is the contact angle between the fluid surface and the container wall.

It is known that no regularity holds in case $\theta+2 \beta<\pi$, hence only the borderline case $\theta+2 \beta=\pi$ remains open.
\end{abstract}

We here want to examine the regularity of solutions of capillary surface type equations (subject to contact angle boundary conditions) on domain $\Omega \subset \boldsymbol{R}^{2}$ in a neighbourhood of a point of $\partial \Omega$ where there is a corner.

To be specific let $\Omega$ (as depicted in the diagram) be a region contained in $D_{R}=\left\{x \in \boldsymbol{R}^{2}:|x|<R\right\} \quad(R>0$ given) such that $\partial \Omega$ consists of a circular segment of $\partial D_{R}$ together with two compact Jordan ares $\gamma_{1}, \gamma_{2}$ such that $\gamma_{1} \cap \gamma_{2}=\{0\} . \gamma_{1}, \gamma_{2}$ are supposed to be $C^{1, \alpha}$ for some $0<\alpha<1$, and to meet at 0 with angle (measured in $\Omega$ ) $\theta, 0<\theta<\pi$. We also suppose (without loss of generality, since we can always take a smaller $R$ ) that $\gamma_{i}$ intersects $\partial D_{o}$ in a single point for each $i=1,2,0<\rho<R$.

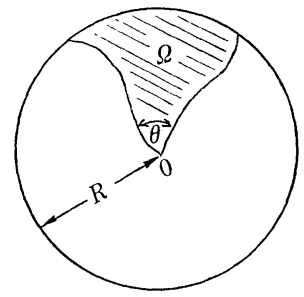

Then we look at (weak) $C^{1, \alpha}(\bar{\Omega} \sim\{0\})$, solutions of the equation

$$
\sum_{i=1}^{2} D_{i}\left(\frac{D_{i} u}{\sqrt{1+|D u|^{2}}}\right)=H(x, u) \text { on } \Omega \text {, }
$$

where $H$ is a locally bounded measurable function on $\bar{\Omega} \times \boldsymbol{R}$.

It is assumed that a contact angle boundary condition holds; to be precise, we suppose 


$$
\nu(X) \cdot \mu(X)=\cos \beta
$$

at each point $X=(x, u(x))$ with $x \in\left(\gamma_{1} \cup \gamma_{2}\right) \sim\{0\}$. Here and subsequently $\nu(X)$ denotes the upward unit normal of the graph $M$ of $u$ at $X$ (although we will assume that $\nu$ is defined on all of $(\bar{\Omega} \sim$ $\{0\}) \times R$ by $\nu(x, t) \equiv(-D u(x), 1) / \sqrt{1+|D u|^{2}}$ for $(x, t) \in(\bar{\Omega} \sim\{0\}) \times R$; thus $\nu$ is constant on vertical lines), and $\mu(X)$ denotes the inward pointing unit normal of the boundary cylider $\left(\left(\gamma_{1} \cup \gamma_{2}\right) \sim\{0\}\right) \times \boldsymbol{R}$. Notice that of course $(0.2)$ can be expressed as $\partial u / \partial \eta / \sqrt{1+|D u|^{2}}=\cos \beta$, where $\partial u / \partial \eta$ denotes the directional derivative of $u$ in the direction of the outward unit normal to $\partial \Omega \sim \partial D_{R}$.

As is well-known, in case $H(x, u) \equiv \kappa u+\lambda(\kappa, \lambda$ constants $)$ the equation (0.1) with boundary condition (0.2) is the usual model for the equilibrium free surface of a fluid in a cylindrical container, with side walls including $\left(\gamma_{1} \cup \gamma_{2}\right) \times \boldsymbol{R}$, subject to the influence of a uniform gravitational field acting in the vertical direction. (The case $\kappa=0$ corresponds to zero gravity, while $\kappa>0, \kappa<0$ correspond to gravitational fields acting vertically downwards and upwards respectively.)

The "contact angle" $\beta$ of (0.2) is supposed to be a constant, with

$$
0<\beta<\pi,
$$

but we could, without significant changes to the proofs, allow the case when $\beta$ is a Hölder continuous function satisfying (0.3) at each point of $\gamma_{1} \cup \gamma_{2}$.

The angle $\theta$ (measured in $\Omega$ ) between the $\operatorname{arcs} \gamma_{1}, \gamma_{2}$ at 0 is assumed to satisfy

$$
0<\theta<\pi, \quad \theta>\pi-2 \widetilde{\beta}
$$

where $\widetilde{\beta}=\beta$ if $0<\beta \leqq \pi / 2$ and $\widetilde{\beta}=\pi-\beta$ in case $\pi / 2<\beta<\pi$. That some condition on the relation between $\theta$ and $\beta$ is necessary in order to deduce any regularity of $u$ near 0 is evident from the results of Concus and Finn [4], who show that, in case

$$
\lim _{t \rightarrow-\infty} \sup _{x \in \Omega} H(x, t)=-\infty \text { and } \lim _{t \rightarrow+\infty} \inf _{x \in \bar{\Omega}} H(x, t)=+\infty,
$$

$u$ is bounded near 0 if and only if $\theta \geqq \pi-2 \widetilde{\beta}$.

The main result to be proved here is given in the following theorem. Notice that we need to assume a-priori that $u$ is bounded in $\Omega$.

THeOREM 1. Suppose $u \in C^{1, \alpha}(\bar{\Omega} \sim\{0\}) \cap L^{\infty}(\Omega)$ satisfies (0.1), (0.2), and suppose that (0.3) and (0.4) also hold.

Then $\lim _{x \rightarrow 0, x \in \bar{\Omega}} u(x)$ and $\lim _{x \rightarrow 0, x \in \bar{\Omega}} D u(x)$ both exist (with values in 
$\boldsymbol{R}$ and $\boldsymbol{R}^{2}$ respectively); thus $u$ extends to a $C^{1}(\bar{\Omega})$ function.

In view of the result of Concus and Finn referred to above, we are able to state the following corollary of the theorem.

Corollary 1. Suppose $u \in C^{1, \alpha}(\bar{\Omega} \sim\{0\})$ satisfies (0.1), (0.2) and suppose (0.3), (0.4), (0.5) also hold.

Then the conclusion of Theorem 1 remains valid.

The general idea of the proof of Theorem 1 is first to show that there is a point $\left(0, z_{0}\right) \in\{0\} \times R$ at which the graph $M$ of $u$ has a nonvertical tangent plane $z=z_{0}+\sum_{i=1}^{2} a_{i} x_{i}$ ( $a_{1}, a_{2}$ constants), in the sense that $\left|u\left(x_{1}, x_{2}\right)-z_{0}-\sum_{i=1}^{2} a_{i} x_{i}\right|=o\left(\sqrt{x_{1}^{2}+x_{2}^{2}}\right)$ as $\sqrt{x_{1}^{2}+x_{2}^{2}} \rightarrow 0$. This is achieved in $\S \S 1-3$, using some geometric measure theoretic arguments (involving interior regularity and first variation theory). A key point here is a positive lower bound for the two dimensional density of $M=$ graph $u$ at any point of $\bar{M} \cap\{0\} \times \boldsymbol{R}$. (See inequality (1.12) of §1.) In particular there are no "cusp-like" singularities. The angle condition (0.4) is needed to prove this lower density bound; (0.4) is not needed for any of the other results in this paper.

Having established the existence of a nonvertical tangent plane at $\left(0, z_{0}\right)$ one then uses (in $\left.\S 4\right)$ the interior regularity theory and the boundary regularity results of Jean Taylor [10], away from $\{0\} \times \boldsymbol{R}$ (i.e., away from the singular part of the boundary cylinder), to conclude the existence of a limit for $D u(x)$ as $x \rightarrow 0$.

We should remark that while this paper is concerned only with nonparametric capillary surfaces in cylindrical containers, it is evident that regularity results for parametric solutions in general polyhedral-type containers satisfying suitable edge and vertex angle conditions can be obtained by appropriate modification of the method described here.

1. Preliminary area bounds. In this section, and subsequently, $\Omega$ and $u$ are as described above, with $\sup _{\Omega}|u| \leqq L<\infty$ ( $L$ a given fixed constant); $\nu$ and $\mu$ are also as described in the introduction, and we use the following additional notation:

$$
\begin{gathered}
D_{\rho}=\left\{x \in \boldsymbol{R}^{2}:|x|<\rho\right\} \quad(\rho>0) ; \\
B_{\rho}(Y)=\left\{X \in \boldsymbol{R}^{3}:|X-Y|<\rho\right\} \quad\left(\rho>0 \text { and } Y \in \boldsymbol{R}^{3}\right)^{1} ; \\
\mu^{(1)}=\lim _{\substack{X \rightarrow 0 \\
X \in \gamma_{1} \times \boldsymbol{R}}} \mu(X), \quad \mu^{(2)}=\lim _{\substack{X \rightarrow 0 \\
X \in \gamma_{2} \times \boldsymbol{R}}} \mu(X) ;
\end{gathered}
$$

${ }^{1}$ As a rule we will represent points in $\boldsymbol{R}^{3}$ by upper-case letters $X, Y, \cdots$ and points in $\boldsymbol{R}^{2}$ by lower-case letters $x, y, \cdots$. 


$$
\begin{aligned}
& M=\operatorname{graph} u=\{X=(x, u(x)): x \in \bar{\Omega} \sim\{0\}\} ; \\
& \partial M=\left\{X=(x, u(x)): x \in \partial \Omega \sim\left(\{0\} \cup \partial D_{R}\right)\right\} ;
\end{aligned}
$$

$\mathfrak{S}^{1}=1$-dimensional Hausdorff measure in $\boldsymbol{R}^{2}$ or $\boldsymbol{R}^{3}$;

$\mathfrak{S}^{2}=2$-dimensional Hausdorff measure in $\boldsymbol{R}^{3}$;

$J$ will denote any constant such that

$$
|H(x, u(x))| \leqq J \text { for all } x \in \Omega \sim\{0\} .
$$

Our first task in this section will be to establish upper bounds on the area of $M$. In fact we will show

$$
\mathfrak{S}_{\mathcal{E}}{ }^{2}\left(M \cap\left(D_{\rho} \times \boldsymbol{R}\right)\right) \leqq c \rho, \quad 0<\rho<R,
$$

where $c$ is a constant depending only on $J, L$ and $R$.

To see this we first multiply the equation (0.1) by a function $\dot{\rho} \in C^{1}(\bar{\Omega} \sim\{0\})$ and integrate over the subdomain $U \equiv\left(D_{o} \sim D_{\sigma}\right) \cap \Omega$, where $0<\sigma<\rho \leqq R$. This gives

$$
-\int_{\tau} \frac{D u \cdot D \dot{\phi}}{\sqrt{1+|D u|^{2}}} d x=\int_{\partial C} \frac{\phi}{\frac{D u \cdot \eta}{\sqrt{1+|D u|^{2}}}} d x+\int_{U} H(x, u) \dot{\phi} d x
$$

where $\eta$ denotes the inward unit normal of $\partial U$. We then take $\dot{\phi} \equiv u$ and let $\sigma \rightarrow 0$. One readily checks that (1.2) then yields (1.1).

We are also here going to need the classical first variation formula for $M$. This says

$$
\int_{M} \delta^{M} \cdot \phi d \mathscr{C}^{2}=-\int_{M} \dot{\phi} \cdot H d \mathscr{S}^{2}-\int_{\partial M} \phi \cdot \eta d \mathscr{S}^{1},
$$

where the notation is as follows:

$\eta$ denotes the unit normal to $\partial M$ which is tangent to $M$ and which points into $\Omega \times \boldsymbol{R}$;

$\boldsymbol{H}=$ mean curvature vector of $M=H(X) \nu(X)$ at each point of $M$ by virtue of (0.1);

$\phi=\left(\phi_{1}, \phi_{2}, \phi_{3}\right)$ is any $C^{1}(\bar{\Omega} \times \boldsymbol{R})$ vector field which vanishes in a neighborhood of $(\{0\} \times \boldsymbol{R}) \cup\left(\partial D_{R} \times \boldsymbol{R}\right) ; \delta^{M} \cdot \dot{\phi}=\sum_{i=1}^{3} \delta_{i}^{M} \dot{\phi}_{i}$, where $\delta M=$ $\left(\delta_{1}^{H}, \delta_{2}^{M}, \delta_{3}^{M}\right)$ is the gradient operator relative to $M$, defined by

$$
\hat{o}_{i}^{\prime \prime} h(X)=\sum_{j=1}^{3}\left(\hat{o}_{i j}-\nu_{i}(X) \nu_{j}(X)\right) D_{j} h(X), \quad X \in M,
$$

whenever $h \in C^{1}(\bar{\Omega} \times \boldsymbol{R})$. (Thus $\delta^{M} h$ is the orthogonal projection of the ordinary gradient $D h(X)$ onto the tangent space of $M$ at $X$.)

Using this formula, we can bound the length of $\partial M$ by the following argument. 
Let $r$ be the radial distance function defined by $r(x, t)=|x|$, $x, t \in \boldsymbol{R}^{2} \times \boldsymbol{R}$, let $\phi$ be any $C^{1}$ vector field on $\bar{\Omega} \times \boldsymbol{R} \sim\{0\} \times \boldsymbol{R}$ with $\sup r|D \phi|<\infty$ and support $|\phi| \subset D_{R} \times \boldsymbol{R}$, and for $0<4 \sigma<\rho<R$ let $\psi_{\sigma} \in C^{1}\left(\boldsymbol{R}^{3}\right)$ be such that $\psi_{\sigma}(x, t)=\gamma(|x|)$ for $(x, t) \in \boldsymbol{R}^{2} \times \boldsymbol{R}$, where $\gamma \in C^{1}(\boldsymbol{R})$ satisfies the conditions:

$$
\left\{\begin{array}{c}
\gamma=0 \text { on }[0, \sigma], \quad \gamma \equiv 1 \text { on }[\rho-\sigma, R] \\
\gamma^{\prime}=\rho^{-1} \text { on }[2 \sigma, \rho-2 \sigma], \quad 0 \leqq \gamma^{\prime} \leqq \rho^{-1} \text { on }[0, R] .
\end{array}\right.
$$

(Thus $\gamma(t) \rightarrow \min \{t / \rho, 1\}$ uniformly as $\sigma \rightarrow 0$ for $t \in[0, R]$.)

Then, upon substituting $\psi_{o} \dot{\phi}$ in place of $\dot{\phi}$ in (1.3) and letting $\sigma \rightarrow 0$, we deduce

$$
\begin{gathered}
\rho^{-1} \int_{M \Gamma\left(D_{\rho} \times \boldsymbol{R}\right)} \rho^{\cdot} \cdot \delta^{M} r d \mathscr{S}^{2}+\int_{\partial \cdot M} \min \{r / \rho, 1\} \dot{\phi} \cdot \eta d \mathfrak{Y}^{1} \\
=-\int_{M} \min \{r / \rho, 1\}\left(\delta^{M} \cdot \dot{\rho}+H \nu \cdot \phi\right) d \mathscr{S}^{2}
\end{gathered}
$$

Now

$$
\eta=\frac{\mu-(\nu \cdot \mu) \nu}{|\mu-(\nu \cdot \mu) \nu|}=\frac{\mu-\cos \beta \nu}{|\mu-\cos \beta \nu|} \text { on } \partial M
$$

by virtue of (0.2). Thus if $\gamma$ is the unit vector bisecting the angle $\theta$ formed by the tangents to $\gamma_{1}, \gamma_{2}$ at 0 , we have

$$
\eta \cdot \gamma \geqq \frac{\mu \cdot \gamma-|\cos \beta|}{|\mu-\cos \beta \nu|} \geqq \frac{1}{2}\left(\sin \frac{\theta}{2}-|\cos \beta|\right)>0
$$

on $\partial M \cap\left(D_{\rho_{0}} \times \boldsymbol{R}\right)$ for sufficiently small $\rho_{0}>0$. (That $\sin \theta / 2-$ $|\cos \beta|>0$ is just a restatement of $(0.4)$.)

By (1.1) we thus deduce from (1.4) (after taking $\phi=$ scalar function $\times \gamma$ and letting $\rho \downarrow 0)$ that

$$
\mathfrak{S}^{1}\left(\partial M \cap\left(D_{R} \times \boldsymbol{R}\right)\right)<\infty .
$$

In terms of the varifold $V=v(M)$ associated with $M([1,3.5])$, this, along with (0.1) and (1.1), tells us that

$$
\|\delta V\|\left(\left(D_{R} \sim\{0\}\right) \times R\right)<\infty,
$$

where $\delta V$ denotes the first variation of $V$ and $\|\delta V\|$ is its total variation $([1,4.1,4.2])$. We can therefore use $[2,3.1(7)]$ to deduce

$$
\rho^{-1} \int_{M \cap\left(D_{\rho} \times \boldsymbol{R}\right)}\left|\delta^{M} r-D r\right|^{2} d \mathscr{S}^{2} \longrightarrow 0 \quad \text { as } \rho \longrightarrow 0 \text {. }
$$

In view of (1.1) (1.9) and Schwartz inequality, we see from (1.4) that 


$$
\begin{aligned}
& \rho^{-1} \int_{M \cap\left(D_{\rho} \times R\right)} \psi \gamma \cdot D r d \mathfrak{S}^{2}+\int_{\partial M} \psi \gamma \cdot \eta d \mathfrak{Y}^{1} \\
& \leqq(1+J) \int_{M}\left(\psi+\left|\delta^{M} \psi\right|\right) d \mathfrak{S}^{2}+o(1)
\end{aligned}
$$

as $\rho \rightarrow 0$, where $\gamma$ is the constant vector of (1.6), and suppose $\psi \subset D_{\rho_{0}} \times \boldsymbol{R}$.

Since $\gamma \cdot D r \geqq \cos \theta / 2>0$, and since (1.6) holds, we then have

$$
\begin{gathered}
\limsup _{\rho \downarrow 0} \rho^{-1} \int_{M \cap\left(D_{\rho} \times \boldsymbol{R}\right)} \psi d \mathscr{S}^{2}+\int_{\partial M} \psi d \mathfrak{S}^{2} \\
\leqq c(1+J) \int_{M}\left(\psi+\left|\delta^{M} \psi\right|\right) d \mathscr{S}^{2}
\end{gathered}
$$

whenever support $\psi \subset D_{\rho_{0}} \times \boldsymbol{R}$, where $c$ depends on $\theta$ and $\beta$. In terms of the varifold $V=\boldsymbol{v}(\boldsymbol{M})$ this says

$$
\|\delta V\|(\psi) \leqq c(1+J) \int\left(\psi+\left|\delta^{M} \psi\right|\right) d\|V\|
$$

by $[2,3.1(2)]$.

With the help of the isoperimetric inequality [1, 7.1] and a minor variation of the iteration argument of [1, 7.5(6)] (taking $f=1$ there), we then deduce

$$
\begin{gathered}
\mathscr{S C}^{2}\left(M \cap B_{\rho}(Y)\right) \geqq c \rho^{2}\left(1+\rho_{0}\right)^{-2}, \quad 0<\rho<\rho_{0}-\sigma, \\
Y \in \bar{M} \cap\left(D_{o} \times R\right)
\end{gathered}
$$

for some positive constant $c$ depending only on $J$ and the constant $c$ in (1.11). We deduce particularly that the bound (1.12) holds also for $Y \in \bar{M} \cap(\{0\} \times \boldsymbol{R})$. For convenience of notation we will henceforth suppose $0 \in \bar{M} \cap(\{0\} \times \boldsymbol{R})$ (this can be arranged by replacing $u$ by $u-z_{0}$ for suitable $z_{0}$ ), and hence (1.12) holds with $Y=0$.

Notice that (1.12) says in particular that $M$ cannot have a "cusp-like" singularity at a point of $\{0\} \times \boldsymbol{R}$. If the condition (0.4) is violated however, it appears intuitively evident that there exists graphs $M$ of bounded mean curvature which do exhibit such singularities.

2. Monotonicity and consequences. In this section we first want to establish a certain monotonicity property. (See (2.6) below.) It seems likely that this can be proved by modifying the relevant argument of Jean Taylor [10]. It will be convenient here however to use standard varifold theory $[1, \S \S 3,4,5.1-5.4]$; the reader will see that only a few of the more elementary aspects of [1] are used in this section, and as in $\S 1$ only the stationary character of $M$, rather than a minimizing property, is needed. 
To begin, suppose $\phi$ is a $C^{1}$ vectorfield in $R^{3}$ with the properties

$$
\begin{aligned}
& \phi \text { is parallel to }(0,0,1) \text { on }\{0\} \times \boldsymbol{R}, \phi \text { is tangent to } \\
& \left(\partial \Omega \sim \partial D_{R}\right) \times \boldsymbol{R} \text { on }\left(\partial \Omega \sim \partial D_{R}\right) \times \boldsymbol{R} .
\end{aligned}
$$

Let $F=\left\{(x, t): x \in \gamma_{1} \cup \gamma_{2} \sim\{0\}, t \leqq u(x)\right\}$ and for $0<\sigma<R$ let $F_{\sigma}=F \cap\{(x, t): \sigma \leqq|x| \leqq R-\sigma\}$. The classical divergence theorem (e.g., $[7,5.6 .9]$ ), which we apply to $F_{\sigma}$ and let $\sigma \rightarrow 0$, gives

$$
\delta W(\psi \phi)=-\int_{\partial . M} \psi \dot{\phi} \cdot \gamma d \mathscr{S}^{1}
$$

whenever $\psi$ is a $C_{c}^{1}\left(D_{R} \times \boldsymbol{R}\right)$ function. Here $W$ denotes the two dimensional varifold $v(F)$ associated with $F$, and $\gamma$ denotes the unit normal of $\partial M$ which is tangent to $F$ and which points into $F$.

Since $\cos \beta \gamma \cdot \phi=\eta \cdot \phi$ ( $\eta$ as in (1.3)) whenever $\phi$ is as in (2.1), we can then multiply by- $\cos \beta$ in (2.2) and add the result to (1.3) (which says $\delta V(\psi \dot{\phi})=-\int_{\partial M} \psi \eta \cdot \phi d \mathfrak{S}^{1}-\int_{M} \psi H \dot{\phi} \cdot \nu d \mathfrak{S}^{2}$ ), thus obtaining

$$
(\delta V-\cos \beta \delta W)(\psi \dot{\phi})=-\int_{M} H \psi \dot{\phi} \cdot \nu d \mathscr{S}^{2}
$$

whenever $\phi$ is as in (2.1). Similarly if we take $\widetilde{W}=\boldsymbol{v}(\widetilde{F}), \quad \widetilde{F}=$ $\left\{(x, t): x \in \gamma_{1} \cup \gamma_{2} \sim\{0\}, t \geqq u(x)\right\}$, we deduce

$$
(\delta V+\cos \beta \delta \widetilde{W})(\psi \dot{\psi} \dot{\phi})=-\int_{M} H \psi \dot{\psi} \cdot \nu d \mathscr{S}^{2} .
$$

Since $\gamma_{1}, \gamma_{2}$ are $C^{1, \alpha}$ curves, one can readily check that there is a $C^{1}$ vector field $\dot{\rho}$ as in (2.1) such that

$$
\begin{aligned}
& \sup _{X \in D_{R} \times \boldsymbol{R}}|X|^{-1-\alpha}|X-\phi(X)|<\infty, \\
& \sup _{x \in D_{R} \times \boldsymbol{R}}|X|^{-\alpha}|D(X-\phi(X))|<\infty .
\end{aligned}
$$

Next, let $Z=V-\cos \beta W$ in case $\cos \beta<0$ and $Z=V+\cos \beta \widetilde{W}$ in case $\cos \beta>0$. By (2.3), (2.3) and (2.4) we then have

$$
|\delta Z(\gamma(|X|)) X| \leqq c \int\left(|X|^{\alpha} \gamma(|X|)+|X|^{1+\alpha} \gamma^{\prime}(|X|)\right) d\|Z\|,
$$

where $c$ depends only on $J$, for any $C_{c}^{1}((-R, R))$ function $\gamma$. In view of this, a minor modification of the argument of $[1,5.1]$ or $[8, \S 3]$ shows that, for a suitable constant $c$,

$$
\exp \left(c \rho^{\alpha}\right) \frac{\|Z\|\left(B_{\rho}(0)\right)}{\rho^{2}} \text { is increasing in } \rho, 0<\rho<R \text {. }
$$

Furthermore, by (1.12), (2.2), (2.6) and [1, 4.12] we deduce that 
there is nonzero stationary varifold $C$ in the varifold tangent of $Z$ at 0 . Thus, writing $\mu_{r}$ to represent the homothetic transformation $X \mapsto r X \quad(r>0)$, we can find a sequence $r_{k} \rightarrow \infty$ so that $V_{\infty}=$ $\lim _{k \rightarrow \infty} \mu_{r_{k^{\sharp}}} V, W_{\infty}=\lim _{k \rightarrow \infty} \mu_{r_{k_{k}}} W$, and $\widetilde{W}_{\infty}=\lim _{k \rightarrow \infty} \mu_{r_{k^{\ddagger}}} \widetilde{W}$ all exist and so that $C=V_{\infty}-\cos \beta W_{\infty}$ or $C=V_{\infty}+\cos \beta \widetilde{W}_{\infty}$ according as $\cos \beta$ is negative or positive. Evidentally $\mu_{r \sharp}\|C\|=\|C\|$ (by (2.6)).

An immediate consequence of (1.12) is that, for each $\rho>0$, there is a sequence $\varepsilon_{k} \rightarrow 0$ such that

$$
B_{\rho}(0) \cap M_{k} \subset\left\{Y \in B_{\rho}(0): \operatorname{dist}\left(Y, \operatorname{spt}\left\|V_{\infty}\right\|\right)<\varepsilon_{k}\right\} .
$$

Here $M_{k}=\mu_{r_{k}}(M)$ and $\operatorname{spt}\left\|V_{\infty}\right\|$ denotes the support of the measure $\left\|V_{\infty}\right\|\left(\left\|V_{\infty}\right\|=\right.$ weight of $\left.V_{\infty}[1,3.1]\right)$.

Indeed, if (2.7) were false, there would exist $\varepsilon>0$, a subsequence $\left\{k^{\prime}\right\} \subset\{k\}$ and a sequence $\left\{X_{k^{\prime}}\right\}$ with $X_{k^{\prime}} \in M_{k^{\prime}} \cap A_{\varepsilon}$ for $k^{\prime}$, where for each $n>0$ we let

$$
A_{\eta}=\left\{Y \in \bar{B}_{\rho}(0): \operatorname{dist}\left(Y, \operatorname{spt}\left\|V_{\infty}\right\|\right) \geqq \eta\right\} .
$$

Applying the inequality (1.12) to $M_{k^{\prime}}$ (notice that (1.12) holds with the same constant $c$ if $M$ is replaced by $M_{k}$, because $M_{l_{c}}=\mu_{r_{k}}(M)$ ), we deduce

$$
\mathfrak{S}_{\mathcal{C}}^{2}\left(M_{k} \cap A_{\varepsilon / 2}\right) \geqq \mathfrak{S}^{2}\left(M_{k} \cap B_{\varepsilon / 2}\left(X_{k}\right)\right) \geqq c \varepsilon^{2} / 4,
$$

thus contradicting the fact that

$$
\limsup _{k \rightarrow \infty} \mathfrak{S}^{2}\left(M_{k} \cap A_{\varepsilon / 2}\right) \leqq\left\|V_{\infty}\right\|\left(A_{\varepsilon / 2}\right)(=0)
$$

(which holds because $\boldsymbol{v}\left(M_{k_{k}}\right) \rightarrow V_{\infty}$ ).

3. Tangent plane for $M$ at 0 . From the interior nonparametric regularity theory $[9, \S 3]$ (alternatively from the parametric theory of $[1, \S 8]$ or [3] or [6]), we deduce that there exist $\lambda, \delta \in(0,1)$ and a constant $c>0$, all depending only on $\rho J$, such that, whenever $Y \in M$ and $B_{\rho}(Y) \cap(\partial \Omega \times \boldsymbol{R})=\varnothing$

$$
B_{\lambda, \rho}(Y) \cap M \text { is connected, }|\nu(X)-\nu(\bar{X})| \leqq c\left(\rho^{-1}|X-\bar{X}|\right)^{j},
$$

for $X, \bar{X} \in B_{2 \rho}(Y) \cap M$.

Let $\left\{r_{k}\right\}$ be the sequence used to construct the varifold $C$ in $\S 2$, let $\Omega_{k}=\left\{r_{k} x: x \in \Omega\right\}, M_{k}=\mu_{r_{k}}(M)$ (=graph $u_{k}$, where $u_{k}$ is defined by $\left.u_{k}(x)=r_{k} u\left(r_{k}^{-1} x\right), x \in \Omega_{k}\right)$, and let $V_{\infty}, W_{\infty}, \widetilde{W}_{\infty}$ be as in $\S 2$. Also, let $\Omega_{\infty}$ be the domain enclosed by the rays which are tangent to $\gamma_{1}$, $\gamma_{2}$ at 0 , so that the Lebesgue measure of $\left[\left(\Omega_{\infty} \sim \Omega_{k}\right) \cup\left(\Omega_{k} \sim \Omega_{\infty}\right)\right] \cap D_{o}$ converges to zero as $k \rightarrow \infty$ for each $\rho>0$.

In view of (3.1) and in view of the fact that (by (0.1)) $M_{k}$ )) $M_{k}$ has mean curvature bounded by $J / r_{k}$, we deduce that 


$$
V_{\infty} L\left(\Omega_{\infty} \times \boldsymbol{R}\right)=\boldsymbol{v}\left(M_{\infty}\right)
$$

where $M_{\infty}$ (=lim $M_{k}$ taken in $\Omega_{\infty} \times \boldsymbol{R}$ in the varifold sense) is either empty or a smooth minimal (not necessarily connected) submanifold of $\Omega_{\infty} \times R$ with

$$
\mathfrak{S}^{2}\left(M_{\infty} \cap B_{\rho}(0)\right)<\infty \quad \text { for each } \rho>0(\text { by }(2.6))
$$

and with $\mu_{r}\left(M_{\infty}\right)=M_{\infty}$ for each $r>0$. This last property just says that $M_{\infty}$ is a cone, which is true by (2.6) and [1, 5.2(2)(a)].

One now readily checks (from the fact that $M_{\infty}$ is a $C^{2}$ cone with zero mean curvature) that

$$
M_{\infty}=\bigcup_{j=1}^{N} \pi_{j} \cap\left(\Omega_{\infty} \times \boldsymbol{R}\right),
$$

where $\pi_{j}$ are planes through the origin and $\pi_{i} \cap \pi_{j} \cap \Omega_{\infty} \times \boldsymbol{R}=\varnothing$ for $i \neq j$. We must consider the possibility that $N=\infty$ here, but in any case by (3.2) we see immediately that at most a finite subcollection of $\left\{\pi_{1}, \pi_{2}, \cdots\right\}$ intersects a given compact subset of $\Omega_{\infty} \times \boldsymbol{R}$. Evidently, since $M_{\infty}$ is the limit (taken in $\Omega_{\infty} \times \boldsymbol{R}$ in the varifold sense) of the sequence $M_{k}$ of graphs, we easily deduce from (3.3) that either

Case 1. $\quad N=1$ and $M_{\infty}=\pi_{1} \cap\left(\Omega_{\infty} \times \boldsymbol{R}\right)$ for some plane $\pi_{1}$ such that $\pi_{1} \cap(\{0\} \times \boldsymbol{R})=\{0\}$; or

Case 2. $\quad N<\infty$ and $M_{\infty}=\bigcup_{j=1}^{N} \pi_{j} \cap\left(\Omega_{\infty} \times \boldsymbol{R}\right)$, where $\pi_{1}, \pi_{2}, \cdots, \pi_{N}$ are planes with the line $\{0\} \times \boldsymbol{R}$ in common. (Notice that to get $N<\infty$ here, it is necessary to use (3.2).)

To proceed further, we need to consider the variational problem satisfied by $M$. For any bounded Borel set $A \subset \boldsymbol{R}^{3}$ and any open $W \subset \Omega \times \boldsymbol{R}$ we let

$$
\begin{aligned}
E(W, A)= & \mathscr{S}^{2}(\partial W \cap \Omega \times \boldsymbol{R} \cap A) \\
& -\cos \beta \mathscr{S C}^{2}(\partial W \cap \partial \Omega \times \boldsymbol{R} \cap A)+\int_{A \cap W} K(X) d X,
\end{aligned}
$$

where $K$ is defined on $\Omega \times \boldsymbol{R}$ by $K(x, t)=H(x, u(x)),(x, t) \in \Omega \times \boldsymbol{R}$, so that $K$ is constant on vertical lines.

We claim that $U=\{(x, t) \in \Omega \times R$ : $t<u(x)\}$ minimizes $E$ in the sense that

$$
E\left(U, B_{\rho}(0)\right) \leqq E\left(W, B_{\rho}(0)\right)
$$

whenever $W$ satisfies 


$$
\begin{array}{cc}
W \subset \Omega \times \boldsymbol{R}, & \mathfrak{S}^{2}\left(\partial W \cap B_{\rho}(0)\right)<\infty, \\
((W \sim U) \cup(U \sim W)) \cap B_{\rho}(0) \subset \subset B_{\rho}(0) .
\end{array}
$$

To see this, first note that the equation (0.1) can be written $\operatorname{div} \nu=K$ on $\Omega \times \boldsymbol{R}$, where $K$ is as above. An alternative way of writing this is

$$
d\left(^{*} \nu\right)=K d x_{1} \wedge d x_{2} \wedge d x_{3} \text { on } \Omega \times \boldsymbol{R},
$$

where ${ }^{*} \nu$ denotes the 2 -form $\nu_{1} d x_{2} \wedge d x_{3}-\nu_{2} d x_{1} \wedge d x_{3}+\nu_{3} d x_{1} \wedge d x_{2}$. Let $[W],[U]$ denote the 3-currents obtained by integrating 3-forms over $W$ and $U$ respectively; $\partial[W], \partial[U]$ are rectifiable in $B_{p}(0)$ by (1.1), (3.5) and [5, 4.5.6(1)].

Next let $\psi_{o}$ be a nonnegative $C^{1}\left(\boldsymbol{R}^{3}\right)$ function with $\psi_{o} \equiv 1$ or $B_{\rho}(0) \sim\left(D_{\sigma} \times \boldsymbol{R}\right), \psi_{\sigma} \equiv 0$ or $D_{\sigma / 2} \times \boldsymbol{R}$ and $\sup _{R^{3}}\left|D \psi_{\sigma}\right| \leqq 3 / \sigma$, and use the identity

$$
\left.\left.\partial([W]-[U])\left(\psi_{\sigma} . *^{*} \nu\right)\right)=([W]-[U])\left(d\left(\psi_{\sigma} . *^{*} \nu\right)\right)\right) .
$$

Letting $\sigma \downarrow 0$ and using $[5,4.5 .6(4)]$ to evaluate the left side of this identity, we deduce

$$
\begin{aligned}
\int_{U \cap B_{\rho}(0)} K(X) d X+\int_{\partial U \cap B_{\rho}(0)} \nu \cdot \eta_{U} d \mathscr{S}^{2} \\
=\int_{W \cap B_{\rho}(0)} K(X) d X+\int_{\partial W \cap B_{\rho}(0)} \nu \cdot \eta_{W} d \mathscr{Y}^{2},
\end{aligned}
$$

where $\eta_{V}, \eta_{W}$ denote the exterior normals of $U$ and $W$ respectively. (See $[5,4.5 .5]$ for the definition of $\eta_{W}$; notice that unless $W$ is a reasonably nice set, we may have $\eta_{W}=0$ on a set of positive $\mathfrak{S}^{2}$ measure in $\partial W \cap B_{\rho}(0)$.)

Since $\eta_{U}=\nu$ on $\partial U \cap(\omega \times \boldsymbol{R})$ and

$$
\eta_{W}=\mu \quad \mathscr{S}^{2} \text {-a.e. on } \partial W \cap(\partial \Omega \times \boldsymbol{R}) \cap\left\{X \in B_{\rho}(0): \eta_{W}(X) \neq 0\right\} \text {, }
$$

we then have (3.4), as required, by virtue of (0.2).

Now define, for any open $W \subset \Omega_{k} \times \boldsymbol{R}$ and any bounded Borel set $A \subset \boldsymbol{R}^{3}$,

$$
\begin{aligned}
E_{k}(W, A)= & \mathscr{S}^{2}\left(\hat{\partial} W \cap\left(\Omega_{k} \times \boldsymbol{R}\right) \cap A\right)-\cos \beta \mathscr{S}^{2}\left(\partial W \cap\left(\partial \Omega_{k} \times \boldsymbol{R}\right) \cap A\right) \\
& +r_{k}^{-1} \int_{W \cap A} K\left(r_{k}^{-1} X\right) d X .
\end{aligned}
$$

(We also include $k=\infty$ in this definition, in which case the last term is to be interpreted as zero.) Since $E_{k}\left(\mu_{r_{k}} W, \mu_{r_{k}} A\right)=r_{k}^{2} E(W, A)$ whenever $W$ is as in (3.5), it is evident from (3.4) that for $k=$ $1,2, \cdots$ we have 


$$
E_{k}\left(U_{k}, B_{\rho}(0)\right) \leqq E_{k}\left(W, B_{\rho}(0)\right)\left(U_{k}=\mu_{r_{k}}(U)\right),
$$

whenever $W$ is an open set such that

$$
\begin{aligned}
& W \subset \Omega_{k} \times \boldsymbol{R}, \quad \mathscr{S}^{2}\left(\partial W \cap B_{\rho}(0)\right)<\infty, \\
& \left(\left(U_{k} \sim W\right) \cup\left(W \sim U_{k}\right)\right) \cap B_{\rho}(0) \subset \subset B_{\rho}(0) .
\end{aligned}
$$

We can now show that $M_{\infty} \neq \dot{\phi}$. In fact we will show that

$$
V_{\infty} L\left(\partial \Omega_{\infty} \times \boldsymbol{R}\right)=0,
$$

which is a stronger statement because $V_{\infty} \neq 0$ by (1.12).

To prove (3.7) first note that since $V_{\infty}=\lim _{k \rightarrow \infty} \mu_{r_{k} \sharp} V$, by virtue of $(1.11)$ and $(2.6)$ we can apply $[1,5.4]$ to deduce that $\Theta^{2}\left(\left\|V_{\infty}\right\|,(Y \geqq 1\right.$ for $\left\|V_{\infty}\right\|-$ a.e. $Y$. If (3.7) fails we can therefore take a point $Y \in \partial \Omega_{\infty} \times \boldsymbol{R} \sim\left((\{0\} \times \boldsymbol{R}) \cup\left(\bigcup_{j=1}^{N} \pi_{j}\right)\right)$ such that $\Theta^{2}\left(\left\|V_{\infty}\right\|, Y\right) \geqq 1$.

Hence for each $\varepsilon>0$ we can find $\rho>0$ such that

$$
\begin{gathered}
B_{2 \rho}(Y) \cap\left((\{0\} \times \boldsymbol{R}) \cup\left(\bigcup_{j=1}^{N} \pi_{j}\right)\right)=\dot{\phi}, \\
\frac{\mathfrak{S}^{2}\left(B_{\rho / 2}(Y) \cap M_{k}\right)}{\pi(\rho / 2)^{2}} \geqq 1-\varepsilon
\end{gathered}
$$

for all sufficiently large $k$, and (by virtue of (2.7))

$$
M_{k} \cap B_{\rho}(Y) \subset\left\{X \in \Omega_{k} \times \boldsymbol{R}: \operatorname{dist}\left(X, \partial \Omega_{k} \times \boldsymbol{R}\right)<\sigma_{k} \rho\right\},
$$

where $\sigma_{k} \rightarrow 0$ as $k \rightarrow \infty$.

Next, let $\left\{f_{k}\right\}$ be a sequence of $C^{\infty}$ mappings of $\boldsymbol{R}^{3}$ into $\boldsymbol{R}^{3}$ with the properties:

$$
f_{k}\left(\bar{\Omega}_{k} \times \boldsymbol{R}\right) \subset \bar{\Omega}_{k} \times \boldsymbol{R}, \quad f_{k}\left(B_{\rho / 2}(Y)\right) \subset B_{\rho / 2}(Y), \quad f_{k}(X)=X,
$$$$
X \in\left(\boldsymbol{R}^{3} \sim B_{\rho}(Y)\right) \cup\left(\partial \Omega_{k} \times \boldsymbol{R}\right), \quad f_{k}\left(B_{\rho}(Y) \sim B_{\rho / 2}(Y)\right) \subset B_{\rho}(Y) \sim B_{\rho / 2}(Y)
$$

$f_{k}\left\{X \in B_{\rho / 2}(Y): \operatorname{dist}\left(X, \partial \Omega_{k} \times \boldsymbol{R}\right)<\sigma_{k}\right\} \subset B_{\rho / 2}(Y) \cap \partial \Omega_{k} \times \boldsymbol{R}$

$$
\sup _{X \in R^{3}}\left\|D f_{k}(X)\right\| \leqq 1+c \sigma_{k}, \quad c \text { independent of } k .
$$

(It is left to the reader to check that such a sequence exists.)

For each $k$ we now let $U_{k}=\mu_{r_{k}}(U), \widetilde{U}_{k}=$ interior $f_{k}\left(U_{k}\right)$, and we let $E_{k}$ be as in (3.4)'. From construction of the $f_{k}$, we know that for $k=1,2, \cdots$,

$$
E_{k}\left(\widetilde{U}_{k}, B_{\rho / 2}(Y)\right)=0,
$$

$$
E_{k}\left(\widetilde{U}_{k}, B_{\rho}(Y) \sim B_{\rho / 2}(Y)\right) \leqq\left(1+c \sigma_{k}\right)^{2} E_{k}\left(U_{k}, B_{\rho}(Y) \sim B_{\rho / 2}(Y)\right),
$$

and, by virtue of (3.8),

$$
E_{k}\left(U_{k}, B_{\rho / 2}(Y)\right)-(1-\varepsilon-|\cos \beta|) \pi(\rho / 2)^{2}+\widetilde{\sigma}_{k} \geqq 0,
$$


where $\tilde{\sigma}_{k} \rightarrow 0$ as $k \rightarrow \infty$. Combining (3.10), (3.11), we deduce that (for $\varepsilon<1-|\cos \beta|$ and $k$ sufficiently large)

$$
E_{k}\left(\widetilde{U}_{k}, B_{\rho}(Y)\right)<E_{k}\left(U_{k}, B_{\rho}(Y)\right),
$$

and hence, since $f_{k}(X) \equiv X$ for all $X \in R^{3} \sim B_{\rho}(Y)$,

$$
E_{k}\left(\widetilde{U}_{k}, B_{o}(0)\right)<E_{k}\left(U_{k}, B_{o}(0)\right) \quad(\sigma>\rho+|Y|),
$$

thus contradicting $(3.4)^{\prime}$ for all sufficiently large $k$. Thus (3.7) is proved; hence

$$
M_{\infty} \neq \phi \quad \text { and } \quad V_{\infty}=\boldsymbol{v}\left(M_{\infty}\right) .
$$

By virtue of (3.1) and the definition of $U_{k}$ it now readily follows that there is an open $U_{\infty} \subset \Omega_{\infty} \times \boldsymbol{R}$ such that $\partial U_{\infty} \cap\left(\Omega_{\infty} \times \boldsymbol{R}\right)=M_{\infty}$ and $\left(U_{\infty} \sim U_{k}\right) \cup\left(U_{k} \sim U_{\infty}\right)$ has measure locally converging to zero. Furthermore by (3.1), (3.3), (3.4)', (2.7), (3.7) and the fact that $\mu_{r_{k} \sharp} V \rightarrow V_{\infty}$, we easily deduce

$$
E_{\infty}\left(U_{\infty}, B_{\rho}(0)\right) \leqq E_{\infty}\left(W, B_{\rho}(0)\right)
$$

for every open $W$ satisfying

$$
\begin{aligned}
& W \subset \Omega_{\infty}+\boldsymbol{R}, \quad \mathfrak{S \mathcal { }}^{2}\left(\partial W \cap B_{\rho}(0)\right)<\infty, \\
& \left(\left(W \sim U_{\infty}\right) \cup\left(U_{\infty} \sim W\right)\right) \cap B_{\rho}(0) \subset \subset B_{\rho}(0) .
\end{aligned}
$$

Here we use the notation that

$$
E_{\infty}(W, A)=\mathfrak{S}^{2}\left(\partial W \cap\left(\Omega_{\infty} \times \boldsymbol{R}\right) \cap A\right)-\cos \beta \mathfrak{S}^{2}\left(\partial 亠 \cap\left(\partial \Omega_{\infty} \times \boldsymbol{R}\right) \cap A\right)
$$

for any $W$ as in (3.14) and any bounded Borel set $A$.

Now we want to show Case 2 is impossible. To see this, note first that in Case $2 U_{\infty}=U_{\infty}^{(1)} \times \boldsymbol{R}$ for some open $U_{\infty}^{(1)} \subset \Omega_{\infty}$ with $\partial U_{\infty}^{(1)}$ a finite union of rays emanating from the origin. Define

$$
E_{\infty}^{(1)}(W)=\mathscr{S}^{1}\left(\partial W \cap \Omega_{\infty} \cap D_{1}\right)-\cos \beta \mathcal{S}^{1}\left(\partial W \cap \partial \Omega_{\infty} \cap D_{1}\right)
$$

for any open $W$ satisfying

$$
\begin{gathered}
W \subset \Omega_{\infty}, \quad \mathfrak{S}^{1}\left(\partial W \cap D_{1}\right)<\infty, \\
\left(\left(W \sim U_{\infty}^{(1)}\right) \cup\left(U_{\infty}^{(1)} \sim W\right)\right) \cap D_{1} \subset \subset D_{1},
\end{gathered}
$$

and note that it follows from (3.13) that

$$
E_{\infty}^{(1)}\left(U_{\infty}^{(1)}\right) \leqq E_{\infty}^{(1)}(W)
$$

for any $W$ as in (3.15).

Since $\Omega_{\infty} \sim \bar{U}_{\infty}^{(1)}$ clearly satisfies a variational principle similar to that satisfied by $U_{\infty}^{(1)}$ but with $\pi-\beta$ in place of $\beta$, in case $N>1$ 
we can suppose without loss of generality that there is a component $W^{*}$ of $U_{\infty}^{(1)}$ with $\bar{W}^{*} \cap \partial \Omega_{\infty}=\{0\}$. But then

$$
E_{\infty}^{(1)}\left(\left(U_{\infty}^{(1)} \sim W^{*}\right) \cup \widetilde{W}^{*}\right)<E_{\infty}^{(1)}\left(U_{\infty}^{(1)}\right),
$$

where $\widetilde{W}^{*}$ is obtained by "smoothing out" the vertex of $W^{*}$ at 0 . Since this contradicts (3.16), we deduce $N=1$.

To show that we also get a contradiction in Case 2 if $N=1$, we note that if $\beta_{0}$ is the angle formed by $U_{\infty}^{(1)}$ at 0 , and if $\beta_{0}<\beta$, then we have

$$
E_{\infty}^{(1)}\left(W^{*}\right)<E_{\infty}^{(1)}\left(U_{\infty}^{(1)}\right)
$$

if $W^{*}$ is constructed as follows:

Let $p \in \partial D_{1 / 2} \cap\left(\partial U_{\infty}^{(1)} \sim \partial \Omega_{\infty}\right)$ and let $q$ be the point on $\partial U_{\infty}^{(1)} \cap \partial \Omega_{\infty}$ at distance $\varepsilon$ from 0 . We then let $W^{*}=U_{\infty}^{(1)} \sim H$, where $H$ is the closed 1/2-plane with $0 \in H \sim \partial H$ and $\{p, q\} \subset \partial H$. For $\varepsilon$ small enough one then easily checks that (3.17) holds. Thus we deduce

$$
\beta_{0} \geqq \beta \text {. }
$$

However, again using the fact that $\Omega_{\infty} \sim \bar{U}_{\infty}^{(1)}$ satisfies a similar variational problem with $\pi-\beta$ in place of $\beta$, we can deduce by the same argument that

$$
\theta-\beta_{0} \geqq \pi-\beta
$$

Adding (3.18) and (3.18) we have $\theta \geqq \pi$, thus contradicting (0.4).

Thus Case 2 is impossible, and we are left with Case 1. Notice that the plane $\pi_{1}$ in Case 1 is uniquely determined by $\beta$ and $\Omega_{\infty}$. In fact a standard (nonparametric) argument (based on the fact that (3.13) holds) shows that $\pi_{1}$ must make an angle (measured in $U_{\infty}$ ) of $\beta$ with each component of $\left(\partial \Omega_{\infty} \times \boldsymbol{R}\right) \sim(\{0\} \times \boldsymbol{R})$. Thus $\pi_{1}$ is characterized by saying that $\pi_{1}$ has a unit normal $\nu^{0}$ with the properties

$$
\nu^{0} \cdot(0,0,1)>0, \quad \nu^{0} \cdot \mu^{(1)}=\cos \beta=\nu^{0} \cdot \mu^{(2)}\left(\mu^{(i)}=\lim _{\substack{X \rightarrow 0 \\ X \in \gamma_{i}}} \mu(X)\right) .
$$

(This characterizes $\pi_{1}$ completely because $\mu^{(1)}$ and $\mu^{(2)}$ are linearly independent.)

Thus we have shown that $M_{\infty}=\pi_{1} \cap\left(\Omega_{\infty} \times \boldsymbol{R}\right)$ with $\pi_{1}$ having unit normal $\nu^{0}$ as in (3.19), independent of the particular sequence $\left\{r_{k}\right\}$ chosen to construct $M_{\infty}$. It follows that $\left\{\mu_{r_{k}} V\right\}$ converges to the same limit $\boldsymbol{v}\left(\pi_{1} \cap\left(\Omega_{\infty} \times \boldsymbol{R}\right)\right)$ for every sequence $r_{k} \rightarrow \infty$. In particular we may take $r_{k}=2^{k}$. One easily checks that (2.7) then implies 


$$
\lim _{\substack{x \rightarrow 0 \\ x \in \Omega}} \frac{\left|u(x)-\sum_{i=1}^{2}\left(\nu_{i}^{0} / \nu_{3}^{0}\right) x_{i}\right|}{|x|}=0,
$$

where $\nu_{1}^{0}, \nu_{2}^{0}, \nu_{3}^{0}$ are the components of the vector $\nu^{0}$ normal to $\pi_{1}$. In particular, we deduce $\lim _{x \rightarrow 0, x \in \Omega} u(x)$ exists, thus completing the proof of the first assertion of Theorem 1 .

4. Conclusion of proof. Here let $M_{k}=\mu_{2^{k}} M, u_{k}(x)=2^{k} u\left(2^{-k} x\right)$, $x \in \Omega_{k l}$, where $\Omega_{k}=\mu_{2^{k}}(\Omega)$. (Thus $M_{k}, \Omega_{k}$ are as in the previous section, with $r_{k}=2^{k}$.)

We know from (3.20) that

$$
\left|u_{k}(x)-\sum_{i=1}^{2}\left(\nu_{i}^{0} / \nu_{3}^{0}\right) x_{i}\right| \longrightarrow 0 \quad \text { as } \quad k \longrightarrow \infty
$$

uniformly for $1 \leqq|x| \leqq 2$.

On the other hand (3.1), applied to $M_{k}$, gives us $\lambda, \delta \in(0,1)$ and $c>0$ so that

$$
\left|\nu^{(k)}(X)-\nu^{(k)}(Y)\right| \leqq c\left(\frac{|X-Y|}{\sigma}\right)^{\delta}
$$

whenever $X=\left(x, u_{k}(x)\right), \quad Y=\left(y, u_{k}(y)\right)$ are such that $|X-Y|<\lambda \sigma$ and $x, y \in\left\{z \in \Omega_{k}: \operatorname{dist}\left(z, \partial \Omega_{\infty}\right)>\sigma\right\}$. Here $\nu^{(k)}$ denotes the upward unit normal of graph $u_{k}$, and $\sigma>0$ is arbitrary.

By combining (4.1), (4.2) we then easily deduce that $D u_{k}(x) \rightarrow$ $\left(\nu_{3}^{0}\right)^{-1}\left(\nu_{1}^{0}, \nu_{2}^{0}\right)$ as $k \rightarrow \infty$, the convergence being uniform for $x \in\left\{y \in \boldsymbol{R}^{2}\right.$ : $\left.1 \leqq|y| \leqq 2, \operatorname{dist}\left(y, \partial \Omega_{\infty}\right)>\sigma\right\}(\sigma>0$ arbitrary).

Writing this last conclusion in terms of $u$, we have

$$
\lim _{\substack{x \rightarrow 0 \\ x \in S_{\sigma}}} D u(x)=\left(\nu_{3}^{0}\right)^{-1}\left(\nu_{1}^{0}, \nu_{2}^{0}\right),
$$

where $S_{\sigma}=\left\{x \in \Omega: \operatorname{dist}\left(x /|x|, \partial \Omega_{\infty}\right)>\sigma\right\}$.

On the other hand, if we use the boundary regularity theory of J. Taylor [10], we deduce by (4.1) that (4.2) actually holds for any $X=\left(x, u_{k}(x)\right), Y=\left(y,{ }_{k}(y)\right)$ with $|X-Y|<\sigma$ and $x, y \in\left\{z \in \Omega_{k}\right.$ : $\left.1 \leqq|z| \leqq 2, \operatorname{dist}\left(z, \Omega_{\infty}\right)<\sigma\right\}$, provided $\sigma$ is sufficiently small (independent of $k$ ). Combining this fact with (4.1) and reasoning as before, we deduce

$$
\lim _{\substack{x \rightarrow 0 \\ x \in T_{o}}} D u(x)=\left(\nu_{3}^{0}\right)^{-1}\left(\nu_{1}^{0}, \nu_{2}^{0}\right)
$$

where $T_{\sigma}=\left\{x \in \Omega\right.$ : $\left.\operatorname{dist}\left(x /|x|, \partial \Omega_{\infty}\right)<\sigma\right\}$.

Theorem 1 is now established by combining (4.3) and (4.4). 


\section{REFERENCES}

1. W. K. Allard, On the first variation of a Varifold, Annals of Math., 95 (1972), 417491.

2. - On the first variation of a Varifold: Boundary behavior, Annals of Math., 101 (1975), 418-446.

3. F. J. Almgren, Jr., Existence and regularity almost everywhere of solutions to elliptic variational problems with constraints, Mem. Amer. Math. Soc., 4, No. 165, (1976).

4. P. Concus and R. Finn, On capillary free surfaces in a gravitational field, Acta Math., 132 (1974), 207-223.

5. H. Federer, Geometric Measure Theory, Springer-Verlag-Berlin-Heidelberg-New York, 1969.

6. E. Giusti, Minimal surfaces and functions of bounded variation, Notes on Pure Mathematics, Australian National University, (1977).

7. W. Klingenberg, A Course in Differential Geometry, Springer-Verlag, Berlin-Heidelberg-New York, 1978.

8. J. Michael and L. Simon, Sobolev and mean-value inequalities on generalized submanifolds of $\mathbf{R}^{n}$, Comm. Pure App. Math., 26 (1973), 361-379.

9. L. Simon, A Holder estimate for quasiconformal maps between surfaces in Euclidean space, Acta Math., 139 (1977), 19-51.

10. J. Taylor, Boundary regularity for solutions to various capillarity and free boundary problems Comm. in P.D.E. 2 (1977), 323-357.

Received March 26, 1979.

University of Melbourne

Parkville, Victoria, 3052 Australia 



\section{PACIFIC JOURNAL OF MATHEMATICS}

\section{EDITORS}

DONALD BABBITT (Managing Editor)

University of California

Los Angeles, CA 90024

Hugo Rossi

University of Utah

Salt Lake City, UT 84112

C. C. MOORE and ANDREW OGG

University of California

Berkeley, CA 94720
J. DUGUNDJI

Department of Mathematics

University of Southern California

Los Angeles, CA 90007

R. FinN and J. Milgram

Stanford University

Stanford, CA 94305

\section{ASSOCIATE EDITORS}
E. F. BECKENBACH
B. H. NeumanN
F. WOLF
K. YoSHIDA

\section{SUPPORTING INSTITUTIONS}

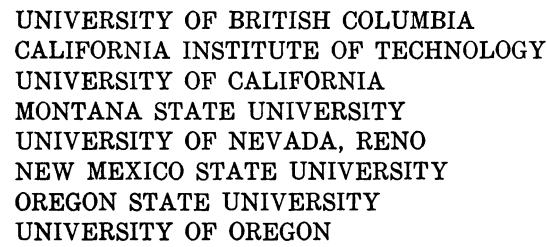

UNIVERSITY OF BRITISH COLUMBIA CALIFORNIA INSTITUTE OF TECHNOLOGY UNIVERSITY OF CALIFORNIA MONTANA STATE UNIVERSITY UNIVERSITY OF NEVADA, RENO NEW MEXICO STATE UNIVERSITY OREGON STATE UNIVERSITY UNIVERSITY OF OREGON

\author{
UNIVERSITY OF SOUTHERN CALIFORNIA \\ STANFORD UNIVERSITY \\ UNIVERSITY OF HAWAII \\ UNIVERSITY OF TOKYO \\ UNIVERSITY OF UTAH \\ WASHINGTON STATE UNIVERSITY \\ UNIVERSITY OF WASHINGTON
}

The Supporting Institutions listed above contribute to the cost of publication of this Journal, but they are not owners or publishers and have no responsibility for its content or policies.

Mathematical papers intended for publication in the Pacific Journal of Mathematics should be in typed form or offset-reproduced, (not dittoed), double spaced with large margins. Please do not use built up fractions in the text of the manuscript. However, you may use them in the displayed equations. Underline Greek letters in red, German in green, and script in blue. The first paragraph or two must be capable of being used separately as a synopsis of the entire paper. Please propose a heading for the odd numbered pages of less than 35 characters. Manuscripts, in triplicate, may be sent to any one of the editors. Please classify according to the scheme of Math. Reviews, Index to Vol. 39. Supply name and address of author to whom proofs should be sent. All other communications should be addressed to the managing editor, or Elaine Barth, University of California, Los Angeles, California, 90024.

50 reprints to each author are provided free for each article, only if page charges have been substantially paid. Additional copies may be obtained at cost in multiples of 50 .

The Pacific Journal of Mathematics is issued monthly as of January 1966. Regular subscription rate: $\$ 84.00$ a year (6 Vols., 12 issues). Special rato: $\$ 42.00$ a year to individual members of supporting institutions.

Subscriptions, orders for numbers issued in the last three calendar years, and changes of address shoud be sent to Pacific Journal of Mathematics, P.O. Box 969, Carmel Valley, CA 93924, U.S.A Old back numbers obtainable from Kraus Periodicals Co., Route 100, Millwood, NY 10546.

PUBLISHED BY PACIFIC JOURNAL OF MATHEMATICS, A NON-PROFIT CORPORATION

Printed at Kokusai Bunken Insatsusha (International Academic Printing Co., Ltd.). 8-8, 3-chome, Takadanobaba, Shinjuku-ku, Tokyo 160, Japan. 


\section{Pacific Journal of Mathematics \\ Vol. 88, No. $2 \quad$ April, 1980}

Reinhold Böhme, Stefan Hildebrandt and Engelbert Tausch, The two-dimensional analogue of the catenary ................. 247

Jean Ellen Taylor, Nonexistence of F-minimizing embedded disks ........ 279

Claus Gerhardt, A free boundary value problem for capillary surfaces ..... 285

Enrico Giusti, Generalized solutions for the mean curvature equation . . . . . 297

Jin-Tzu Chen, On the existence of capillary free surfaces in the absence of gravity.................................... 323

Leon M. Simon, Regularity of capillary surfaces over domains with corners.......................................... 363

Nicholas Jacob Korevaar, On the behavior of a capillary surface at a re-entrant corner.................................... 379

Henry Wente, The symmetry of sessile and pendent drops ............ 387

E. Gonzalez, Umberto Massari and I. Tamanini, Existence and regularity for the problem of a pendent liquid drop ...................... 399

Henry Wente, The stability of the axially symmetric pendent drop ........ 421

David Siegel, Height estimates for capillary surfaces . . . . . . . . . . . . 471

Bruce Edward Turkington, Height estimates for exterior problems of capillarity type ................................ 517

Robert Finn, The sessile liquid drop. I. Symmetric case .............. 541 